\title{
Improved Power Aware Location Based Routing
}

\author{
Tarandeep Singh Kang ${ }^{1}$, Maninder Kaur ${ }^{2}$, Dr K V P Singh ${ }^{3}$ \\ ${ }^{1,2,3}$ (ECE, Doaba Institute of Engineering \& Technology/ Punjab Technical University, India)
}

\begin{abstract}
With rapid growth in wireless Ad Hoc networks, Quality of service factor has drawn a great attention of researchers. Whereas network topology of wireless networks constantly changes, resources like bandwidth and energy are also limited. As power is very important factor for the proper working of Ad Hoc networks, need of the time is to research about efficient utilization of power. In the paper, we improve LAR (Location-Aided Routing), one of the most famous location based routing methods, which uses GPS technique to get information about the location of mobile node. Our new protocol considers both areas of routing and power. At first, propose a more efficient routing method which finds more stable paths. Secondly, a power aware method is proposed to have a prior knowledge about the remaining battery backups of all the intermediate nodes so that the nodes with battery backups equal to or greater than the minimum threshold power are the part of path between source and destination. This work presents an efficient approach for providing QOS in power aware scenario.
\end{abstract}

Keywords: Ad hoc networks, Average power, Expected Zone, Requested Zone, Threshold power

\section{INTRODUCTION}

An ad hoc network is a wireless network also known as Infrastructure less network that establish communications without a fixed network infrastructure or any central administration. It is a collection of nodes which are mobile and also have the capability of working as routers. These nodes can be connected in arbitrary manner dynamically. These nodes when work as routers, discovers and maintains routes to the other nodes in the network.

This is the key feature of future wireless networks to adapt and exist without a fixed infrastructure. Ad Hoc networking is helpful in emergency rescue and search operations, meetings in which information has to be shared quickly and in inhospitable terrains for acquisition of data.

The task to effectively route the data packets in terms of energy consumption and bandwidth is very important. For efficient routing, many routing protocols have been proposed. Earlier Reactive Routing protocols were developed which used to flood the routing packets in all the directions without considering the location of the destination node. This resulted in increased bandwidth consumption and large end to end delay, whereas in Table driven routing protocols, each node maintains a Routing table that stores a large amount of information.

This required performing a large amount of computations. Control packets overhead increases which in turn consumed the limited network bandwidth further resulting in premature loss of battery life. Location Aided Routing Protocols were then developed which reduced this bandwidth consumption. To find the direction of propagation of packets, these location based protocols uses the Global Positioning System (GPS). Therefore bandwidth consumption can be decreased by finding the direction of propagation. In this paper, we propose a power aware routing method which helps to find the stable paths in terms of power by utilizing the concept of global location information. The proposed protocol Improved Power Aware Location Based Routing (IPALBR) protocols use location information to minimize the Request Zone to reach the destination node with a path which is alive for the maximum time.

\section{RELATED WORK}

There are a number of location based routing protocols that provide path from source to the destination. Location based routing protocols use the following parameters.

\subsection{EXPECTED ZONE}

Consider, source node $\mathrm{S}$ wants to send the data packet to the destination node $\mathrm{D}$ at time $\mathrm{T}$ [8]. Assume that the location of $\mathrm{D}$ is known to $\mathrm{S}$ at time Ti. Also it knows velocity $\left(\mathrm{V}_{\mathrm{D}}\right)$ of $\mathrm{D}$ with which $\mathrm{D}$ travels. Maximum distance traveled by $\mathrm{D}$ in any direction can be calculated as:

$$
r=V_{D}\left(T-T_{i}\right)
$$




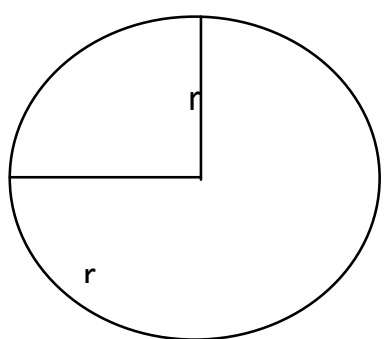

Figure 2.1 Expected Zone

\subsection{REQUEST ZONE}

To find a path from the Source to the destination, the area where the request packets are sent or broadcast is known as a Request Zone. In the traditional routing algorithms, Request Zone is the complete network [8]. For e.g. RREQ packets are broadcasted in all the directions to find the optimal path from source to the destination node in the protocols such as AODV and DSR. LAR tries to minimize the request zone by confining it to the smallest rectangular area which contains both sender and the receiver.

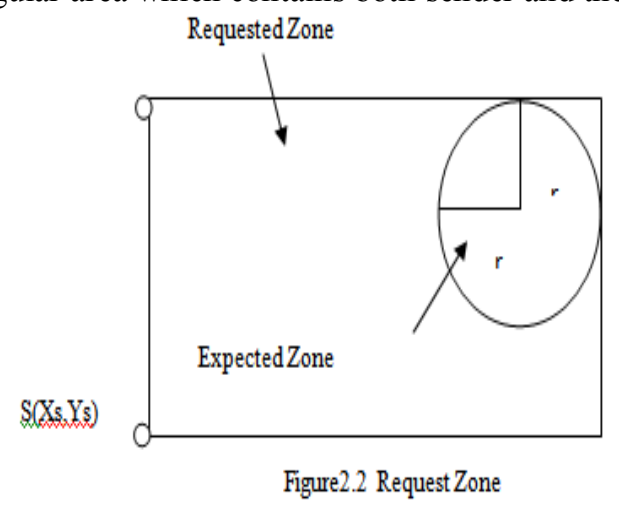

\subsection{Global Position System}

GPS is a system of satellites [2], ground control stations, and receivers that allows users to determine their position. By capturing and storing that position, GPS receivers "digitize" spatial data as they walk, drive, or otherwise traverse the land. Receivers differ in their ability to receive and process GPS signals and users can have a huge affect on accuracy depending on the methods used to collect and process data.

\subsection{Types of Routing Protocol}

Location Based Routing uses the following types of routing protocols.

\subsubsection{Energy Aware Location Based Routing Protocol}

Energy aware location based routing technique use location information by using GPS system and additionally takes a grid method. In this routing protocol each node has GPS in Ad-Hoc network divided by virtual grid [7]. This assumption means that each node knows the location of itself and where they are included in the grid network. To maintaining information of neighborhoods, nodes in each grid communicate each other by beacon message which includes ID, geographical location, and energy. From this communication, node which has the largest energy becomes grid header. And header node is changed when the rest of its energy equals to $1 / 2$ of second node. Grid headers communicate each other by longer period than the one of general node. The data sent by each grid header includes information of all nodes in its grid. So grid header can get the information of entire nodes.

\subsubsection{Improved Location Aided Routing}

ILAR (Improved Location Aided Routing) is another location based technique which uses the concept of base line lying in between the source and destination node. Node which is closest to this line of sight will be chosen as the next intermediate node. As the transmitting node check the distance of every neighboring node from base line and find the closest neighbor for further transmission.

\subsubsection{Location Aware Routing Protocol with Dynamic Adaptation of Request Zone}

Mobile Ad hoc Networks is an on demand routing protocol which decreases the search area given by LAR [9]. In LAR the search area is the smallest rectangle containing both sender as well as receiver. LARDAR 
reduces this rectangle to triangular zone, which helps in reducing the routing overheads. But the calculations done to find whether the node lie in the forwarding zone or not increases the overhead on the node. This results in decrease in battery of the node.

\subsubsection{Location Based Power Aware Routing}

LARDAR used the concept of Triangle zone and the angles $\alpha$ and $\beta$. The protocol LBPAR uses the concept of triangle zone [5]. But instead of using the angular values in route request packet as in LARDAR, this protocol uses the concept of slopes of line which can be calculated using the following formula:

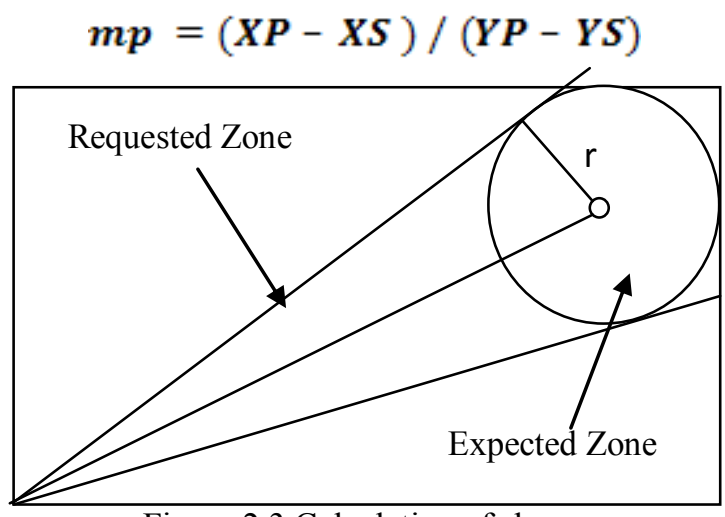

Figure 2.3 Calculation of slopes

The Base objective of LBPAR is to find an optimal path in terms of bandwidth consumption along with the reduction in power loss of a node. Here, the route discovery process starts when source node $\mathrm{S}$ initiates a request to send the data packet to destination node $\mathrm{D} . \mathrm{m} 1$ and $\mathrm{m} 2$ are the slopes of the line. $\mathrm{D}(\mathrm{XT}, \mathrm{YT})$ are the points where the tangents drawn from source to the expected zone touches the expected zone. From the mathematical equations it is clear that $\mathrm{m} 1$ and $\mathrm{m} 2$ will be the maximum and minimum slopes for any line drawn between the source and any point in between these two tangents. These two slopes [1] can be calculated from the following quadratic equation:

$$
m X d-m X s-Y d+Y s=r *\left(1+m^{2}\right)^{1 / 2}
$$

Source node $S$ broadcast a route request packet to find the path. Format of RREQ packet on receiving the RREQ

packet node ' $\mathrm{N}$ ' calculates the slope $m_{n}$ using its own location co-ordinates and source location coordinates. If

its slope lies between the max and min slope i.e. $\left(\mathrm{m} 1 \geq m_{n} \geq \mathrm{m} 2\right)$ then node will again broadcast the packet otherwise node drops the packet. This routing protocol is use to routing packets between mobile nodes in an ad hoc network using the Global Positioning System. Unlike routing protocols like LAR, ILAR, LARDAR this protocol considers various parameters like bandwidth requirement and battery life of all the intermediate nodes on a path to destination. Increase in the battery life tends to high probability of routing path. Based on evaluation of LBPAR is proved to be a better protocol as it helps in lowering the bandwidth consumption of the network and also helps in increasing the battery life by decreasing the number and complexity of calculations.

\subsection{Problem Definition}

In Location Based Power Aware Routing (LBPAR), if the source node wishes to communicate with the destination node, route discovery is initiated. The path is then set up accordingly. In the route discovery process, the packet is sent to only that next node which lies in the minimum and the maximum slopes. This way the path is formed between the source and destination and then information is sent. Nodes coming in the path are taken to form the path. But no focus is given to the remaining battery backups of the nodes. LBPAR helps in reducing the overheads at each node by decreasing the number of calculations performed at each node, which in turn increased the battery life of node. But it can't provide the guarantee to delivery of packet on existing path because no concern is given to the remaining battery backups of nodes. Due to this, there is no idea about what time the path would be alive. This makes the paths found unstable. So there is no guarantee that the sent information packets would reach the destination node or not. 


\section{PROPOSED WORK}

The proposed protocol is an efficient routing scheme which improves the efficiency of location-aided routing (LAR). Proposed approach is an extended version of Location Based power Aware Routing (LBPAR). Some parameters used for the calculation of expected zone and requested zone in this Routing protocol are minimum slope, maximum slope, TTL and minimum threshold power for communication. Route request packet (RREQ) can be sent to the neighbor node based on the minimum, maximum slope and if power of the node is greater than or equal to the threshold power.

\subsection{Threshold Power}

Threshold power is the power set initially which is taken as a reference to select the nodes with power greater than or equal to this reference value. This power is defined initially before the start of the Route Discovery process.

\subsection{Phases of IPALBR}

There are three phase in this protocol

I. Discovery of Route

II. Reply of Route

III. Maintenance of Route

\subsubsection{Discovery of Route}

Source node S (Xs, Ys) wants to communicate with the destination node D (Xd, Yd) in Figure 3.1. The intermediate nodes are 1, 2, 3, 4, 5, 6 and 7. For this communication to be possible first of all Source node $\mathrm{S}$ calculates the expected zone. It uses the destination coordinates to calculate the expected zone. After calculating the expected zone, it uses the expected zone to calculate the requested zone. It is shown in the Figure 3.1. After this, RREQ request is initiated by the source node $S$, this RREQ packet contains the information like TTL, minimum slope, maximum slope, minimum available power, broadcast id, source address, and destination address. The source node collects all this information and then sends the RREQ packet to its neighbors. After receiving the RREQ packet from the source node, the neighbors first of all checks the destination address of the RREQ, The packet is consumed if the destination address is the address of any one of the neighbor node and then RREP packet is sent to the source node.

The value of TTL is checked if the destination address is not equal to the neighbor node address, if the value of TTL is less than or equal to zero the node discards the RREQ packet; else it calculates the slope of that node from the source node. If the slope lies between the maximum slope and the minimum slope, then it attaches its own address to the list of visited nodes and then RREQ packet is forwarded to its neighbor nodes.

The RREQ packet is discarded by the node if the route can qualify neither minimum slope nor maximum slope.

Every node which receives the RREQ packet follows this process until the RREQ packet is successfully received by the destination node.

Figure 3.1 illustrates an example of Discovery of route process of IPALBR which finds the path between source node S and the destination node D. The path between Source node S and destination node D is S-1-2-3-4-5-6-7-D. Algorithm, Algorithm for calculating average power, and flow chart of discovery of route process are shown below.

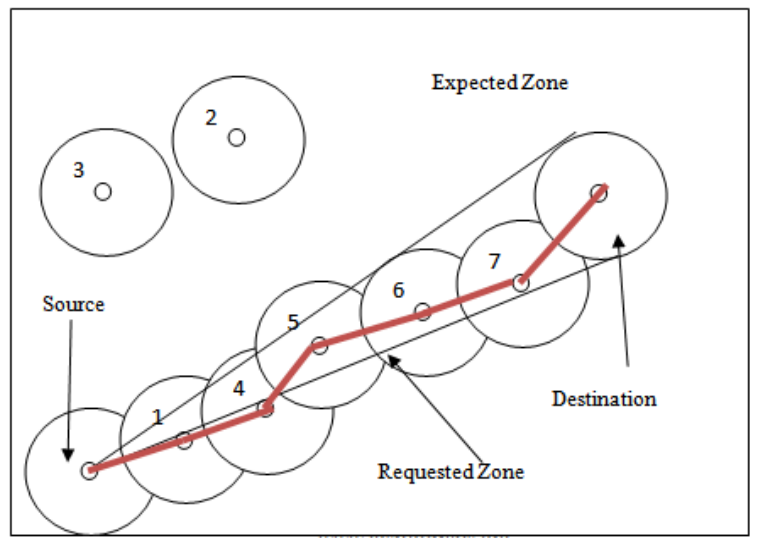

Figure 3.1 Discovery of Route phase of IPALBR 
This process is followed by every node which receive the RREQ packet until the RREQ receive the by destination node. Above figure is an example of route discovery process of IPALBR which find the path between source need $\mathrm{S}$ and destination node $\mathrm{D}$. Source node $\mathrm{S}$ to destination node D path is S-1-2-3-4-5-6-7-D.

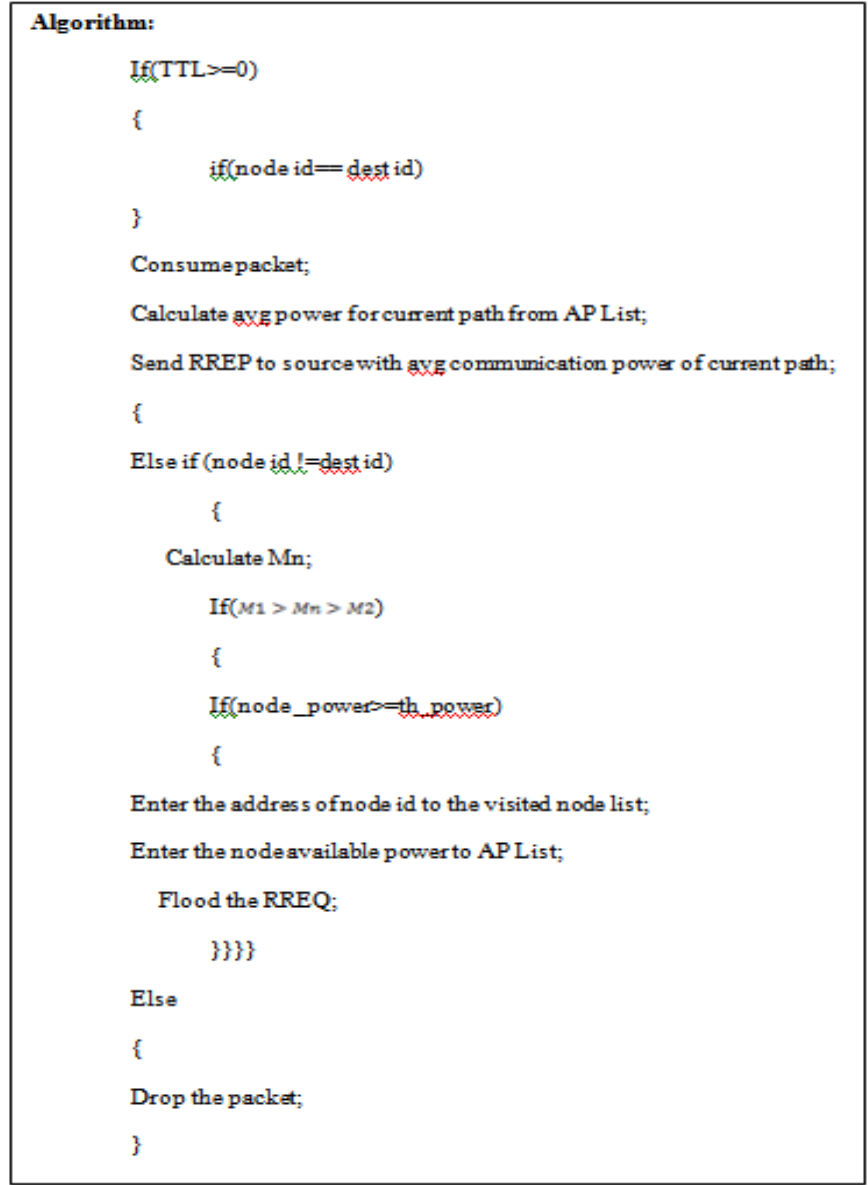

\subsubsection{Reply of Route}

In the reply of route phase the packet header destination address is checked if the destination receives the RREQ packet. If the destination address is the address of node then it checks the minimum and the maximum slopes. RREQ packet is consumed if slope lies between these two slopes and then finds the minimum available power from AP List in a packet header and then sent RREP packet to source node with minimum communication power via same path as the path used to receive RREQ packet from the source to the destination. Otherwise RREQ packet is discarded. The reply of route path from the destination node to the source node D-7-6-5-4-3-2-1-s is illustrated by the figure 3.2.

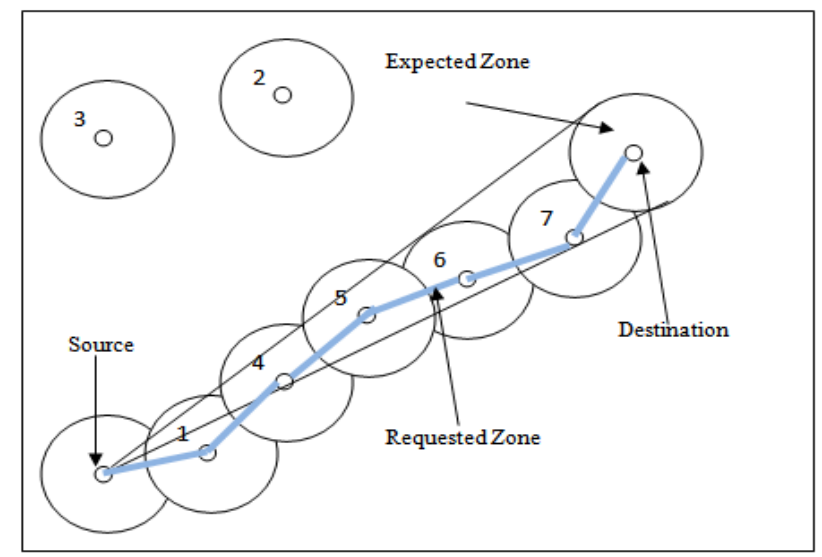

Figure 3.2 Reply of Route phase of IPALBR 


\subsubsection{Maintenance of Route}

There is high mobility of node in the Ad-hoc networks, so the links between various nodes are likely to break. Thus, the routing path has to be maintained. For example node 4 in Figure 3.3 moves outside to the reach of its neighbor, so the route from the source to the destination breaks. Maintenance of route process is used in this case to maintain the route from the source to the destination. In this phase when a node does not receive a RREP packet it will break the path. So in this case, the node sends a route error packet (RERR) to the source node. A new path to the destination node is reconstructed by the Source node after the Source node receives this packet. As Figure 3.3 shows, node 4 moves outside the reach of its neighbors so the path breaks. Then new path is created by using node 3 . So the source to destination path after maintenance is S-1-3-5-6-7-d.

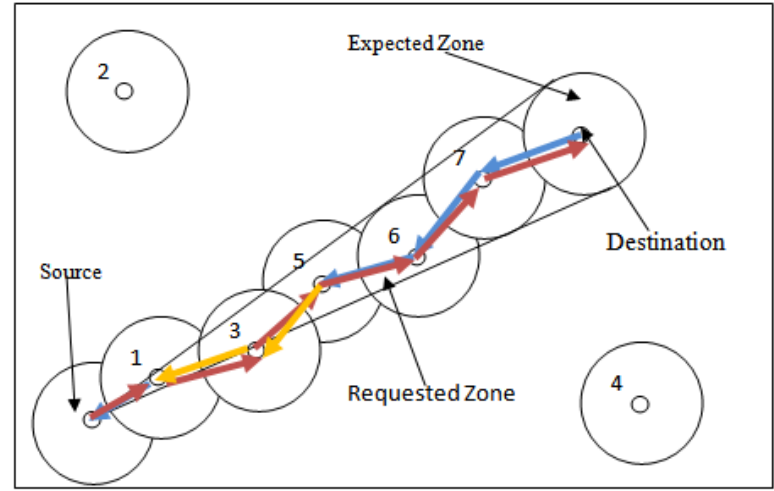

Figure 3.3 Maintenance of Route Phase of IPALBR

\section{COMPARISON WITH LBPAR}

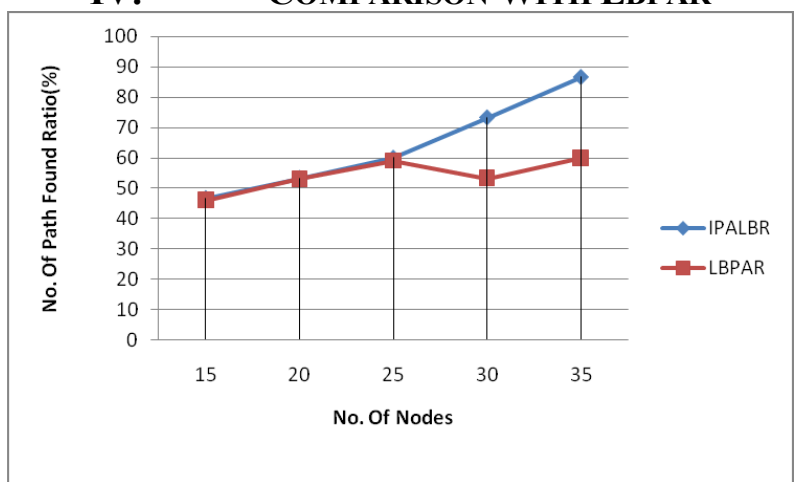

Figure 4.1 Comparison of IPALBR with LBPAR

Figure 4.1 shows the comparison between IPALBR and LBPAR protocol in terms of number of paths found between the source and the Destination. When number of nodes increase then the number of paths found also increases in the IPALBR but in the LBPAR protocol number of paths found remains almost in constant.

Also the number of paths found in the IPALBR protocol are more stable than the LBPAR protocol because in the IPALBR, a threshold value of power is taken by virtue of which only those nodes are included in the path between the source and destination which have battery backup greater than or equal to the threshold value. Due to this the source has the idea, approximately what time the path found would be alive. Depending upon the time that the information packet will take to travel from source node to the destination node, the data is sent. The path is formed only by selecting the nodes with battery backups greater than or equal to the minimum threshold power. The nodes with battery backups less than this threshold value are discarded. Therefore if the path has all the nodes with battery backups greater than or equal to the threshold value, the information is sent and otherwise a new path is searched. But no such approach is used in LBPAR as all the nodes coming in the path from source to destination are made the part of the path. So there is no stability of paths formed. Thus more routes are found in IPALBR; also stability of the routes found is more in IPALBR than in LBPAR. 


\section{CONCLUSION AND FUTURE WORK}

Wireless Ad Hoc networks have several characteristics different from wired networks which make them completely different networks. Whereas network topology of wireless networks constantly changes, resources like bandwidth and energy are also limited. As power is very important factor for the proper working of Ad Hoc networks, need of the time is to research about efficient utilization of power. In the paper, we improve LAR (Location-Aided Routing), one of the most famous location based routing methods, which uses GPS technique to get information about the location of mobile node. Our new protocol considers both areas of routing and power. At first, propose a more efficient routing method which finds more stable paths .Secondly, a power aware method is proposed to have a prior knowledge about the remaining battery backups of all the intermediate nodes so that the nodes with battery backups equal to greater than the minimum threshold power are the part of path between source and destination.

The proposed protocol is an efficient routing scheme which improves the efficiency of location-aided routing (LAR). Proposed approach is an extended version of Location Based power Aware Routing (LBPAR). Some parameters used for the calculation of expected zone and requested zone in this Routing protocol are minimum slope, maximum slope, TTL and minimum threshold power for communication. Route request packet (RREQ) can be sent to the neighbor node based on the minimum, maximum slope and if power of the node is greater than or equal to the threshold power.

On analyzing IPALBR, proposed protocol improves the Quality of Service in terms of power and reduces the RREQ flooding. The proposed protocol uses power awareness factor to provide more stable and efficient path for communication from source to destination mobile node.

"Improved Power Aware Location Based Routing" protocol helps in dealing with the two major quality factors power and RREQ flooding. But the calculations required in this protocol are more as for finding stable paths more time is required, which consumes more battery. Also, nodes with limited power are not used, therefore to find more stable paths, nodes with maximum battery backups are found, which increases delay. This protocol can further be improved by reducing the number of calculations performed, so that more battery power of the nodes is saved.

\section{REFERENCES}

[1] D. D. Perkins and H. D. Hughes, "A survey on quality-of-service support for mobile ad hoc networks," Wireless Communications and Mobile Computing, 2002

[2] G. Dommety and R. Jain. "Potential Networking Applications of Global Positioning System (GPS)," Technical Report TR-24, Computer Science Department, The Ohio State University, April 1999

[3] Jangsu Lee, Seunghwan Y00, and Sungchun Kim," Energy aware Routing in Location based Ad-hoc Networks", Proceedings of the 4th International Symposium on Communications, Control and Signal Processing, ISCCSP 2010, Limassol, Cyprus, 3-5 March 2010

[4] Mohammad A. Mikki," Energy Efficient Location Aided Routing Protocol for Wireless MANETs", (IJCSIS) International Journal of Computer Science and Information Security Vol. 4, No. 1 \& 2, 2009

[5] P.K.Suri Dr. M. K. Soni, Mrs. Parul Tomar" Location Based Power Aware Routing"

[6] Pramita M, Christian P and Shivajit M" Stability Aware Routing: Exploiting Transient Route Availability in MANETs Proceeding HPCC'07 Proceedings of the Third international conference on High Performance Computing and Communications Pages 508520, Springer-Verlag Berlin, Heidelberg (2007

[7] Tzay-Farn Shih, Hsu-Chun Yen, "Location-aware routing protocol with dynamic adaptation of request zone for mobile ad hoc networks", Springer Wireless Netw (2008) 14:321-333,DOI 10.1007/s11276-006-9955-y

[8] Wenzhu Zhang, Jiandong Li and Xuan Wang, "Cost-Efficient QoS Routing Protocol for Mobile Ad Hoc Networks", Proceedings of the 19th International Conference on Advanced Information Networking and Applications (AINA'05).

[9] Y.B. Ko and N. H. Vaidya, "Location-Aided Routing in Mobile Ad Hoc-Scale for Ad Hoc Networks," Proceedings of the second ACM international symposium on Mobile ad hoc networking \& computing, Long Beach, CA, USA, pp. 22-32, October 2001 